\title{
Higher twists in polarized DIS and the size of the constituent quark
}

\author{
A. V. Sidorov \\ Bogoliubov Laboratory of Theoretical Physics, Joint Institute for Nuclear Research, 141980 Dubna, Russia \\ C. Weiss \\ Theory Center, Jefferson Lab, Newport News, Virginia 23606, USA
}

(Received 28 February 2006; published 17 April 2006)

\begin{abstract}
The spontaneous breaking of chiral symmetry implies the presence of a short-distance scale in the QCD vacuum, which phenomenologically may be associated with the size of the constituent quark, $\rho \approx 0.3 \mathrm{fm}$. We discuss the role of this scale in the matrix elements of the twist- 4 and 3 quark-gluon operators determining the leading power $\left(1 / Q^{2}\right)$-corrections to the moments of the nucleon spin structure functions. We argue that the flavor-nonsinglet twist- 4 matrix element, $f_{2}^{u-d}$, has a sizable negative value of the order $\rho^{-2}$, due to the presence of sea quarks with virtualities $\sim \rho^{-2}$ in the proton wave function. The twist- 3 matrix element, $d_{2}$, is not related to the scale $\rho^{-2}$. Our arguments support the results of previous calculations of the matrix elements in the instanton vacuum model. We show that this qualitative picture is in agreement with the phenomenological higher-twist correction extracted from an NLO QCD fit to the world data on $g_{1}^{p}$ and $g_{1}^{n}$, which include recent data from the Jefferson Lab Hall A and COMPASS experiments. We comment on the implications of the short-distance scale $\rho$ for quark-hadron duality and the $x$-dependence of higher-twist contributions.
\end{abstract}

DOI: 10.1103/PhysRevD.73.074016

PACS numbers: 13.60.Hb, 12.38.Lg, 12.39.-x, 13.88.+e

\section{INTRODUCTION}

Polarized deep-inelastic scattering (DIS) has extensively been studied in fixed-target experiments with electron and muon beams [1]. The main goal of such measurements is the extraction of the polarized parton densities in the nucleon from the data in the region of approximate Bjorken scaling, where the $Q^{2}$-dependence is governed by perturbative QCD (DGLAP evolution). However, most of the precise data are at momentum transfers $Q^{2} \sim$ few $\mathrm{GeV}^{2}$, where nonperturbative effects in the $Q^{2}$ dependence (power corrections) generally cannot be neglected. Estimates of these corrections are needed in order to include these data in the QCD analysis. The effect of phenomenological power corrections on the extracted parton densities has been investigated in Refs. [2,3].

With the polarized DIS data becoming more and more precise, the study of the power corrections themselves has emerged as an interesting subject. The $1 / Q^{2}$-corrections to the lowest nonzero moments of the spin structure functions, $g_{1}$ and $g_{2}$, are governed by matrix elements of QCD operators of twist 4 and 3 , describing nonperturbative correlations of the quark and gluon fields in the nucleon [4,5]:

$$
\begin{gathered}
\left\langle N\left|\bar{\psi} \gamma_{\alpha} g \tilde{G}^{\beta \alpha} \psi\right| N\right\rangle=2 f_{2} s^{\beta}, \\
\left\langle N\left|\bar{\psi} \gamma^{\{\alpha} g \tilde{G}^{\beta\} \gamma} \psi\right| N\right\rangle= \\
2 d_{2}\left(p^{\{\alpha} p^{\beta\}} s^{\gamma}-p^{\gamma} p^{\{\beta} s^{\alpha\}}\right) \\
+ \text { traces, }
\end{gathered}
$$

where $\tilde{G}^{\alpha \beta}=(1 / 2) \epsilon^{\alpha \beta \gamma \delta} G_{\gamma \delta}$ is the dual gluon field strength, and $p^{\alpha}$ and $s^{\alpha}$ the nucleon four-momentum and polarization vector; we follow the conventions of
Refs. [6,7]. By extracting the coefficients of the $1 / Q^{2}$ corrections from the data one can thus obtain information about the structure of the nucleon in QCD. The SLAC E155X experiment [8] and the Jefferson Lab Hall A experiment [9] have extracted the twist-3 matrix elements from combined measurements of the structure functions $g_{1}$ and $g_{2}$, and found surprisingly small values, $d_{2} \lesssim 10^{-2}$. Recent analyses have also attempted to extract the twist- 4 matrix element, $f_{2}$, from the power corrections to the first moment of $g_{1}$ [10-13]; see Ref. [14] for a review.

The theoretical estimation of the higher-twist matrix elements (1) and (2) is a challenging problem, requiring a description of the nucleon in terms of QCD degrees of freedom. The key question is which nonperturbative scales govern the quark-gluon correlations measured by the twist4 and 3 operators. This question is intimately related to the role of the "vacuum structure" of QCD in determining the structure of the nucleon.

There is strong evidence for the existence of a shortdistance scale in the QCD vacuum, significantly smaller than the size of the nucleon. It is determined by the characteristic size, $\rho$, of the nonperturbative field configurations instrumental in the spontaneous breaking of chiral symmetry. Numerous observations suggest a "two-scale picture" of hadron structure,

$$
\rho \ll R,
$$

where $R$ is a typical radius of the nucleon (say, the charge radius). Phenomenologically, the short-distance scale may be associated with the "size" of the constituent quark. In fact, the success of effective models based on constituent quark degrees of freedom could not be explained without the hierarchy (3). It is natural to ask what the existence of 
this short-distance scale implies for the quark-gluon correlations probed by the twist -4 and 3 operators.

A microscopic model incorporating the short-distance scale associated with chiral-symmetry breaking is the instanton vacuum, in which the quarks obtain a dynamical mass, $M$, by interaction with a "medium" of instantons of characteristic size $\rho \approx 0.3 \mathrm{fm}$, see Refs. [15,16] for a review. The fundamental assumption of diluteness of the instanton medium (smallness of the packing fraction) implies that, parametrically, $M \ll \rho^{-1}$. Since the range of the chiral forces binding the constituent quarks is of the order $M^{-1}$, this hierarchy translates into a "two-scale picture" of hadron structure, Eq. (3). The higher-twist matrix elements (1) and (2) were calculated in this model in Refs. [17,18]. It was found that the flavor-nonsinglet twist-4 matrix element is determined by the inverse instanton size (i.e., the inverse size of the constituent quark), $f_{2}^{u-d} \sim \rho^{-2}$, and has a sizable negative value. The twist- 3 matrix element, $d_{2}$, however, is parametrically suppressed, $d_{2} \sim(M \rho)^{4}$, in agreement with the experimental data (this prediction was later confirmed by lattice QCD calculations [19]). An interesting question is whether these results depend specifically on the assumption of instantons as the dominant gluonic vacuum fluctuations, or whether they already follow from the more general "two-scale picture" of hadron structure.

In this paper we further explore the connection between the short-distance scale due to chiral-symmetry breaking and the twist-4 and 3 quark-gluon correlations governing the $1 / Q^{2}$-corrections to the nucleon spin structure functions. In Sec. II, we argue that, on general grounds, the flavor-nonsinglet twist-4 matrix element, $f_{2}^{u-d}$, has a sizable negative value of the order $\rho^{-2}$, due to the presence of sea quarks with virtualities $\sim \rho^{-2}$ in the nucleon wave function. The twist-3 matrix element, $d_{2}$, however, is not related to the scale $\rho^{-2}$. Our arguments provide additional insights into the origin of the instanton vacuum results, and suggest that they may be of more general nature. In Sec. III, we present the results of a next-to-leading order (NLO) QCD analysis of the world data for $g_{1}^{p}$ and $g_{1}^{n}$, including recent data from the Jefferson Lab Hall A [20] and COMPASS [21] experiments, in which we extract the flavor-nonsinglet twist-4 matrix element, $f_{2}^{u-d}$. The sign and order-of-magnitude are found to be in agreement with the our qualitative arguments (as well as the instanton vacuum results). This analysis extends previous phenomenological estimates of the higher-twist contribution to polarized DIS by Leader et al. [2,3]. In contrast to other analyses [12] we perform the QCD fit to the data (including higher-twist corrections) for the $x$-dependent structure function, computing the moments only at the last stage, by integration of the fit. Furthermore, we extract directly the isovector (flavor-nonsinglet) twist-4 matrix element, which is practically scheme-independent and thus provides a much cleaner probe of the nonperturbative quark-gluon correlations than the singlet matrix element. Finally, in Sec. IV, we comment on the implications of the "two-scale picture" of hadron structure for the $x$-dependence of the higher-twist contribution and quark-hadron duality in the spin structure functions.

\section{HIGHER-TWIST MATRIX ELEMENTS AND THE SIZE OF THE CONSTITUENT QUARK}

The spontaneous breaking of chiral symmetry implies the existence of a short-distance scale in the QCD vacuum, significantly smaller than the typical hadronic size. It is determined by the characteristic size, $\rho$, of the nonperturbative gluon field configurations which change the quark chirality, see Fig. 1. A gauge-invariant measure of this size is the ratio of the dimension-5 "mixed" quark-gluon condensate to the usual dimension-3 quark condensate,

$$
m_{0}^{2} \equiv \frac{\left\langle\bar{\psi} \sigma^{\alpha \beta} g G_{\alpha \beta} \psi\right\rangle}{\langle\bar{\psi} \psi\rangle}=\frac{2\left\langle\bar{\psi} \nabla^{2} \psi\right\rangle}{\langle\bar{\psi} \psi\rangle} .
$$

Here $\sigma^{\alpha \beta} \equiv(i / 2)\left[\gamma^{\alpha}, \gamma^{\beta}\right]$, and $\nabla_{\alpha} \equiv \partial_{\alpha}-i g A_{\alpha}$ is the covariant derivative. The two representations of the dimension-5 operator are related by the identity

$$
g G_{\alpha \beta}=i\left[\nabla_{\alpha}, \nabla_{\beta}\right],
$$

and the Heisenberg equations of motion for the quark fields. Lattice simulations indicate that $m_{0}^{2} \geqslant 1 \mathrm{GeV}^{2}$ at a normalization point of $\mu \sim 1 \mathrm{GeV}$ [22,23] (substantially larger values were obtained in Ref. [24]). A more precise interpretation of the ratio (5) in terms of a size of field configurations becomes possible with specific assumptions about the shape of these configurations in a given gauge. In the instanton vacuum $[15,16]$, where the chirality-flipping field configurations are (anti-) instantons in singular gauge, one has

$$
m_{0}^{2}=4 \rho^{-2},
$$

at the scale $\mu \sim \rho^{-1}$ [25]. The lattice results are consistent with an average instanton size of $\rho \approx 0.3 \mathrm{fm}$. We note that in the presence of more than one light quark flavor, the chirality flip due to the instanton happens in a manyfermionic interaction ('t Hooft vertex), whence the scale $\rho$ may also be interpreted as the range of chiral-symmetry breaking quark-quark interactions in the QCD vacuum.

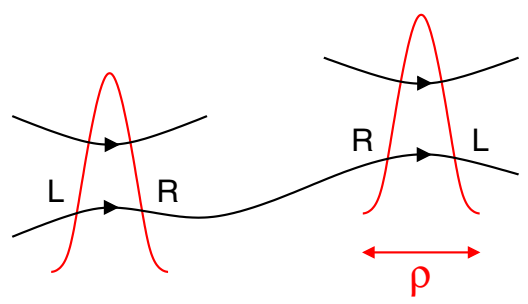

FIG. 1 (color online). Origin of the short-distance scale, $\rho$, associated with chiral-symmetry breaking. 
A simple heuristic argument suggests that the twist-4 matrix element, $f_{2}$, Eq. (1), may be related to the shortdistance scale, $\rho$. Substituting in the twist- 4 operator the gluon field by the commutator of covariant derivatives, Eq. (5), and making use of gamma matrix identities and the Heisenberg equations of motion of the quark fields, one can convert the twist-4 operator to the form [6]

$$
f_{2}: \quad \bar{\psi} \gamma^{\beta} \gamma_{5}\left(-\nabla^{2}\right) \psi
$$

In this form, it can be compared with the axial current operator, which measures the quark contribution to the nucleon spin,

$$
g_{A}: \quad \bar{\psi} \gamma^{\beta} \gamma_{5} \psi
$$

We see that the operator (7) measures the correlation of the spin of the quarks with the square of their canonical momentum. The existence of the short-distance scale, $\rho$, implies that, generally speaking, the nucleon wave function contains "sea" quarks with virtualities (four-momenta squared) up to the scale $\rho^{-2}$. If the spin of these quarks is correlated with the nucleon spin, one would expect the flavor-nonsinglet twist-4 matrix element to be of the order

$$
f_{2}^{u-d} \sim g_{A} \rho^{-2} .
$$

Here we limit ourselves to the flavor-nonsinglet $(u-d)$ operators, which are not affected by the $U(1)_{A}$ anomaly (henceforth, $g_{A}$ denotes the nucleon's isovector axial coupling). Furthermore, if the flavor-nonsinglet sea quarks are polarized along the direction of the nucleon spin, and if the quark canonical momentum squared on average takes positive values, as suggested by Eq. (4), one would conclude that $f_{2}^{u-d}<0$.

The above argument supposes the existence of a dynamical mechanism which polarizes the flavor-nonsinglet sea quarks in the nucleon. In fact, such a mechanism can be found in the effective dynamics resulting from the spontaneous breaking of chiral symmetry. In the large- $N_{c}$ limit of $\mathrm{QCD}$, the effective dynamics at distances $\gtrsim \rho$ can be described by massive "constituent" quarks, coupled to a Goldstone pion field in a chirally invariant way [26,27],

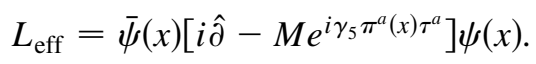

The dynamical quark mass, and the coupling to the pion field, are active for quark virtualities $-k^{2} \leqslant \rho^{-2}$, whence $\rho \ll M^{-1}$ can be interpreted as the size of the constituent quark. The large- $N_{c}$ limit implies a semiclassical description of the nucleon, in which the nucleon is characterized by a classical pion field of size $R \sim M^{-1}$ [28]. Thus, the effective dynamics described by Eq. (10) embodies the "two-scale picture" of the nucleon described in the introduction, Eq. (3).

Consider now the matrix element of the flavornonsinglet axial current operator, i.e., the nucleon's isovector axial charge, in the effective theory of constituent quarks coupled to a pion field, Eq. (10). After appropriate

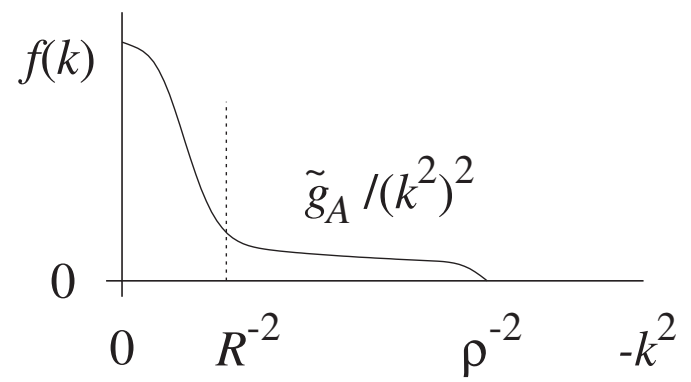

FIG. 2. Distribution of quark virtualities, $k^{2}$, in the proton's isovector axial charge, as given by the effective chiral theory, Eq. (11). The twist- 4 matrix element, $f_{2}^{u-d}$, is of the order of the integral of $k^{2} f(k)$.

projections on nucleon states with definite spin/flavor/momentum quantum numbers, the matrix element can be represented in the form

$$
g_{A}=\int \frac{d^{4} k}{(2 \pi)^{4}} f(k)
$$

where $k$ represents the four-momentum of the quark connected to the axial current operator. We are interested in the distribution of quark virtualities, $k^{2}$, in this integral [29]. This distribution consists of two components, shown schematically in Fig. 2. The bulk of the nucleon's axial charge is carried by valence and sea quarks with virtualities of the order of the size of the nucleon, $-k^{2} \sim R^{-2}$. In addition, however, there is a contribution from sea quarks interacting with the classical pion field, which have virtualities extending up to $-k^{2} \sim \rho^{-2}$. Assuming that $\rho \ll R$, the power behavior and the coefficient of the this largevirtuality "tail" are completely determined by general features of the chiral dynamics. They follow from the leading term in the long-wavelength expansion (gradient expansion) of the quark loop in the background pion field (see Fig. 3):

$$
f(k) \sim \frac{\tilde{g}_{A}}{\left(k^{2}\right)^{2}} \quad\left(-k^{2} \gg R^{-2}\right),
$$

where the coefficient is given by

$$
\tilde{g}_{A} \equiv \frac{4 N_{c} M^{2}}{9} \int d^{3} x \operatorname{tr}\left[-i \tau^{a} U_{\mathrm{cl}}^{\dagger}(x) \partial_{a} U_{\mathrm{cl}}(\boldsymbol{x})\right] .
$$

Here $U_{\mathrm{cl}}(\boldsymbol{x}) \equiv \exp \left[i \pi_{\mathrm{cl}}^{a}(\boldsymbol{x}) \tau^{a}\right]$ denotes the static classical

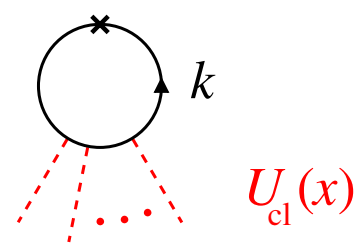

FIG. 3 (color online). The sea quark contribution to the axial charge, giving rise to the large-virtuality tail $\left(-k^{2} \sim \rho^{-2}\right)$ in the distribution $f(k)$, Eqs. (12) and (13). 
pion field in the nucleon rest frame. In fact, the expression (13) coincides with the leading-order gradient expansion of the axial charge induced by the classical pion field (as used e.g. for calculating $g_{A}$ in the Skyrme model). The power behavior (12) implies that the integral (11) depends only logarithmically on the short-distance scale $\rho$ :

$$
g_{A} \sim \log \frac{\rho}{R} .
$$

Thus, $g_{A}$ is dominated by quark virtualities of the order $R^{-2} \ll \rho^{-2}$, and the presence of the large-virtuality tail in the distribution of Fig. 2 is of minor importance.

The presence of the large-virtuality tail in the axial charge distribution becomes crucial, however, for the twist-4 matrix element, $f_{2}^{u-d}$. When passing from QCD to the effective theory of constituent quarks, QCD operators must be "translated" to operators in the effective theory. It is natural to assume that in the case of the twist-4 QCD operator (7) the translation is given by

$$
\left.\left.\bar{\psi} \gamma^{\beta} \gamma_{5}\left(-\nabla^{2}\right) \psi\right|_{\mathrm{QCD}} \rightarrow C \bar{\psi} \gamma^{\beta} \gamma_{5}\left(-\partial^{2}\right) \psi\right|_{\mathrm{eff}},
$$

where $C>0$ is a coefficient of order unity. This was explicitly demonstrated in the instanton vacuum model, where the operator matching follows from the integration over instanton-type gauge field configurations [17]. By analogy with the axial current, the matrix element of the constituent quark operator (15) is now given by the integral of $k^{2}$ times the momentum distribution of $g_{A}$, Eq. (11). This factor suppresses contributions from virtualities $-k^{2} \sim R^{-2}$, and enhances the contributions from the large-virtuality tail. As a result, the twist- 4 matrix element is parametrically of the order

$$
f_{2}^{u-d} \sim \rho^{-2},
$$

with the coefficient proportional to $\tilde{g}_{A}$, in agreement with our estimate (9). Furthermore, since the quark virtualities in the tail of the distribution are spacelike, $k^{2}<0$, we conclude that $f_{2}^{u-d}<0$.

The integral determining $f_{2}^{u-d}$ in the effective chiral theory contains a would-be quadratic divergence, which is cut off at the scale $\rho^{-2}$. While the parametric order and sign of $f_{2}^{u-d}$ follow from general features of the chiral dynamics, the numerical value is very sensitive to the precise way in which the UV cutoff (i.e., the finite size of the constituent quark) is implemented. The instanton vacuum model, which implies a definite form of the UV cutoff resulting from the fermionic zero modes of the instantons [27], and which also allows one to uniquely determine the matching coefficient for the effective twist4 operator, Eq. (15) [17], gives (with $\rho=0.3 \mathrm{fm}$ ) [18]

$$
f_{2}^{u-d} \approx-0.5 g_{A} \rho^{-2}=-0.22 \mathrm{GeV}^{2} .
$$

However, in view of the principal uncertainties in the modeling of the dynamics of quark field modes of virtualities $\sim \rho^{-2}$ this result should be viewed as a rough estimate.
On general grounds, one expects that in QCD with cutoff regularization the matrix elements of twist-4 operators are proportional to the square of the cutoff [30]; this is realized in our approach if the cutoff is identified with the scale $\rho^{-1}$. We do not consider here the logarithmic scale dependence of the twist- 4 matrix element which results from the standard composite operator renormalization [4]; this dependence is much weaker than the principal uncertainty in our estimates of the twist- 4 matrix element.

It is interesting that the estimates of $f_{2}^{u-d}$ obtained in QCD sum rule calculations [31,32] (for a critical discussion of these calculations, see Ref. [33]) agree in sign and order-of-magnitude with our estimate, and with the instanton vacuum result, see Table I. Our estimate disagrees with the bag model [5], which gives a positive result for $f_{2}^{u-d}$ (taking into account the different convention for the sign of $f_{2}$ in that paper). This model, however, does not respect the QCD equations of motion, and therefore cannot claim to give a realistic description of quark-gluon correlations in the nucleon.

When applying the same reasoning as above to the twist3 matrix element, $d_{2}$, we find that after the substitution (5) the quark-gluon operator does not produce a contracted covariant derivative. In this operator, all derivatives are "kinematic," i.e., they are needed to support the spin of the matrix element. This operator does not probe the virtuality of the quarks in the nucleon, and its matrix element does not receive essential contributions from quark virtualities of the order $\rho^{-2}$. Thus, the correlations probed by the twist- 3 matrix element are of essentially different nature from those probed by the twist- 4 one. Beyond this qualitative difference, we see no simple way to estimate the twist- 3 matrix element more accurately on grounds of general features of the effective dynamics alone (see also Ref. [34]). In the instanton vacuum, an additional suppression of $d_{2}$ results from the fact that the coefficient of the corresponding effective operator in the effective chiral theory is parametrically small in the instanton packing fraction (i.e., $d_{2}$ is zero in the single instanton approximation). This suppression appears to be due to the $O(4)$ invariance (in the Euclidean metric) of the instanton field [17]. In a sense, the twist- 3 operator is a much more subtle probe of nonperturbative quark-gluon correlations in the QCD vacuum than the twist-4 operator, whose matrix element can be estimated on general grounds.

TABLE I. Comparison of theoretical estimates of the flavornonsinglet twist-4 proton matrix element, $f_{2}^{u-d}$.

\begin{tabular}{ll}
\hline \hline & $f_{2}^{u-d}\left[\mathrm{GeV}^{2}\right]$ \\
\hline Instantons [17,18] & -0.22 \\
QCD sum rules (Balitsky et al.) [31] & $-0.16 \pm 0.04$ \\
QCD sum rules (Stein et al.) [32] & $-0.06 \pm 0.02$ \\
Bag model [5] & +0.09 \\
\hline \hline
\end{tabular}




\section{TWIST-4 MATRIX ELEMENT FROM A QCD FIT TO POLARIZED DIS DATA}

It is interesting to see to which extent our qualitative conclusions about the higher-twist matrix elements are supported by the experimental data. To this end, we attempt to extract the flavor-nonsinglet twist-4 matrix element, $f_{2}^{u-d}$, from the power corrections to the spin structure functions $g_{1}^{p}$ and $g_{1}^{n}$. To accuracy $1 / Q^{2}$, the tree-level QCD expansion for the first moment of $g_{1}^{p}-$ $g_{1}^{n}$ is given by [4-6]

$$
\begin{aligned}
\int_{0}^{1} d x g_{1}^{p-n}\left(x, Q^{2}\right)= & \frac{a_{0}^{u-d}}{6}+\frac{M_{N}^{2}}{27 Q^{2}} a_{2}^{u-d} \\
& +\frac{4}{27 Q^{2}}\left(M_{N}^{2} d_{2}^{u-d}+f_{2}^{u-d}\right)
\end{aligned}
$$

(when QCD radiative corrections are included, the coefficients acquire a logarithmic $Q^{2}$-dependence). The first term is the leading-twist (LT) contribution, proportional to the matrix element of the flavor-nonsinglet twist-2 spin-1 operator, $a_{0}^{u-d}$, with $a_{0}^{u-d} \equiv g_{A}$ (Bjorken sum rule). The second term represents the target mass corrections (TMC), proportional to the spin-3 twist-2 matrix element, $a_{2}^{u-d}$. The third term is the dynamical higher-twist (HT) contribution, involving the twist- 3 and 4 matrix elements (2) and (1). The twist-3 matrix element, $d_{2}$, has been extracted from independent measurements of the third moment of the spin structure function $g_{2}$ (with the Wandzura-Wilczek contribution subtracted). The SLAC E155X experiment [8] and the recent Jefferson Lab Hall A analysis [9] report values of $d_{2}^{p, n} \leqslant 10^{-2}$, in good agreement with the instanton vacuum prediction [17]. With these values, the contribution of the $M_{N}^{2} d_{2}^{u-d}$ term to the $1 / Q^{2}$ corrections in (18) is more than an order of magnitude smaller than the instanton estimate for $f_{2}^{u-d}$, Eq. (17). This supports the qualitative conclusion from our two-scale picture, that the dominant power corrections are those associated with the short-distance scale, $\rho$. We shall thus neglect the $M_{N}^{2} d_{2}^{u-d}$ term compared to $f_{2}^{u-d}$ in Eq. (18), and ascribe the phenomenological power correction entirely to the twist -4 matrix element. This theoretical simplification will be justified a posteriori by the fact that the numerical value of $f_{2}^{u-d}$ extracted in this way is indeed much larger than the measured $M_{N}^{2} d_{2}^{u-d}$.

The dynamical higher-twist contribution to the $x$-dependent structure functions, $g_{1}^{p}\left(x, Q^{2}\right)$ and $g_{1}^{n}\left(x, Q^{2}\right)$, has been extracted from NLO QCD fits to the world data (see references in Ref. [35]), including the new $g_{1}^{n}$ data from the Jefferson Lab Hall A experiment [20], as well as the deuteron data from COMPASS [21]. These fits are based on the ansatz

$$
g_{1}^{p, n}\left(x, Q^{2}\right)=g_{1}^{p, n}\left(x, Q^{2}\right)_{\mathrm{LT}+\mathrm{TMC}}+\frac{h^{p, n}(x)}{Q^{2}},
$$

where the leading-twist contribution (including target mass corrections) is calculated using the Leader-StamenovSidorov parametrization of the polarized parton densities [35], and $h^{p(n)}$ parametrizes the dynamical higher-twist corrections. In order to extract directly the flavornonsinglet higher-twist correction, we have modified the fit procedure of Refs. [2,35] and parametrized not $h^{p(n)}(x)$ individually, but their difference and sum. The kinematic cuts applied in the new fit are the same as in the fit of Ref. [35], $Q^{2} \geq 1 \mathrm{GeV}^{2}$ and $W^{2} \geq 4 \mathrm{GeV}^{2}$. The results for the difference, $h^{p}(x)-h^{n}(x)$, obtained in this way is shown in Fig. 4.

Integrating the higher-twist contribution over the $x$-range covered in our fit we get

$$
\int_{0.017}^{0.75} d x\left[h^{p}(x)-h^{n}(x)\right]=-0.046 \pm 0.016 \mathrm{GeV}^{2}
$$

If we neglected the contribution from the large- $x$ region (we shall argue in Section IV that this is consistent with our two-scale picture of the structure of the nucleon), as well as the small- $x$ region, and regarded the integral (20) as an estimate of the first moment, we would obtain [cf. Eq. (18)]

$$
f_{2}^{u-d}=-0.31 \pm 0.11 \mathrm{GeV}^{2} .
$$

For a rough data-based estimate of the contribution of the large- $x$ region, we consider the integrals up to $x=1$ computed with a linear extrapolation of the fit into this region, based on the two rightmost data points in Fig. 4, and a constant extrapolation, based on the rightmost data point only (we neglect the small- $x$ contribution). Taking the average of the two integrals as an estimate of the central value, and half the difference as an estimate of the error, we get

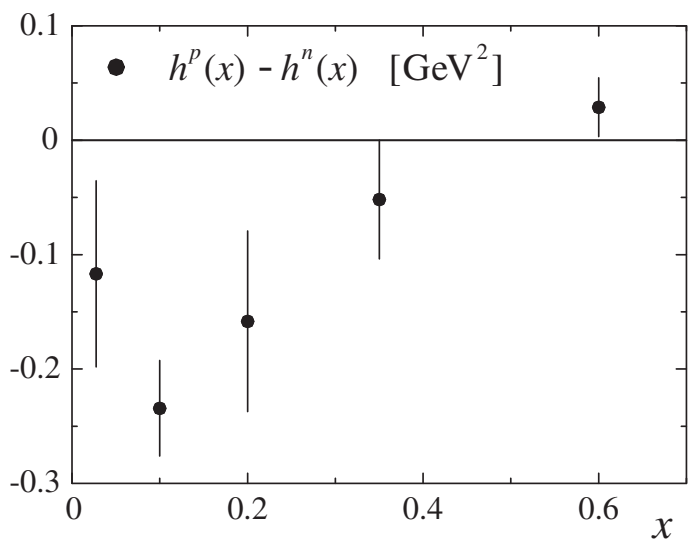

FIG. 4. The difference of higher-twist corrections to the proton and neutron spin structure functions, $h^{p}(x)-h^{n}(x)$, Eq. (19), as extracted from our NLO QCD fit to the world data (see references in Ref. [35]), including recent $g_{1}^{n}$ data from the Jefferson Lab Hall A experiment [20], as well as deuteron data from COMPASS [21]. 


$$
\int_{0}^{1} d x\left[h^{p}(x)-h^{n}(x)\right]=-0.028 \pm 0.019 \mathrm{GeV}^{2},
$$

corresponding to

$$
f_{2}^{u-d}=-0.20 \pm 0.14 \mathrm{GeV}^{2} .
$$

Both estimates (21) and (23) agree in sign and in order-ofmagnitude with our qualitative prediction, Eq. (9), and the result obtained in the instanton vacuum, Eq. (17).

In the QCD fit, the leading-twist parton densities and coefficient functions were taken in the $\overline{\mathrm{MS}}$ scheme. The studies of Ref. [2] found that the higher-twist corrections to $g_{1}$ in different factorization schemes (in particular, in the $\overline{\mathrm{MS}}$ and JET schemes) coincide within errors. Note also that Eq. (21) agrees well with our previous estimate based on the JET scheme [36]. In that estimate, also preliminary HERMES data on the deuteron structure function were taken into account [37]. The recent analysis of Ref. [38] shows that inclusion of the new HERMES proton and deuteron data [39] does not substantially change the higher-twist contribution, whence we have not explicitly included these data in our present analysis.

Our result for $f_{2}^{u-d}$ agrees well with that obtained by Deur et al. [12] in a recent analysis of power corrections to the Bjorken sum rule (their $f_{2}^{p-n} \equiv \frac{1}{3} f_{2}^{u-d}$ in our conventions). Our method differs from that of Ref. [12] in that we perform the QCD fit to the data (including higher-twist corrections) for the $x$-dependent structure function, computing the moments only at the last stage, by integration of the fit. Nevertheless, the results for the higher-twist contribution from both approaches are comparable, which is very encouraging. Our result disagrees in sign with that obtained in an earlier analysis of the $Q^{2}$-dependence of the Bjorken sum rule [40], which combined an empirical parametrization of the electroproduction cross section in the resonance region with the QCD parametrization in the DIS region, see also Refs. [41,42].

\section{DISCUSSION AND OUTLOOK}

In our discussions so far we considered the twist-4 correction to the first moment of $g_{1}(x)$, which is related to the matrix element of the local twist-4, spin-1 operator, $f_{2}$. Much more information is contained in the $x$-dependence of the higher-twist contribution. A detailed study of the $x$-dependence of the twist- 4 contribution on the basis of chiral dynamics and the instanton vacuum is beyond the scope of the present paper. Here we would like to offer only some general comments on this problem.

The idea of a two-scale picture of the nucleon outlined in Section I, cf. Eq. (3) can also be expressed in a partonic language. In this formulation, constituent quarks/antiquarks appear as correlations in the transverse spatial distribution of quarks/antiquarks and gluons, with a transverse size, $\rho$, significantly smaller than the transverse size of the fast-moving nucleon, $R$. (This formulation is in fact used to discuss the effect of the constituent quark structure of the nucleon on high-energy $p p$ scattering with multiple hard processes [43].) This picture applies to average values of the quark/antiquark longitudinal momentum fraction, $x \lesssim 0.5$, for which the transverse size of configurations in the nucleon wave function is of the order of the typical hadronic size. In this formulation, the twist- 4 correction to the spin structure function can be related to the average transverse momentum squared of the polarized quarks/ antiquarks in the nucleon $[44,45]$. Extending the reasoning of Section II, one would argue that due to chiral dynamics the flavor-nonsinglet polarized sea quark distribution involves transverse momenta squared of the order $k_{\perp}^{2} \sim \rho^{-2}$. This suggests an interesting connection between the flavornonsinglet twist- 4 corrections to $g_{1}$ and the large flavor asymmetry of the twist- 2 sea quark distribution, $\Delta \bar{u}(x)-$ $\Delta \bar{d}(x)$, predicted by chiral dynamics in the large- $N_{c}$ limit [46]. The result of our QCD fit, Fig. 4, indicates that the twist-4 correction is indeed localized at relatively small values of $x \sim 0.1$, supporting the connection with the sea quark distribution. A similar connection between the flavor asymmetry and the presence of large transverse momenta in the sea quark distributions was noted in Ref. [47], where instanton-induced sea quark components in the nucleon wave function were considered in a phenomenological model with no reference to the large- $N_{c}$ limit.

The existence of the short-distance scale due to chiralsymmetry breaking also has some interesting qualitative implications for quark-hadron duality in polarized DIS. The two-scale picture of the structure of the nucleon, cf. Eq. (3), implies a parametric classification of the hadronic excitation spectrum of the nucleon. Nucleon resonances such as the $\Delta$ are excitations with energies (in the CM frame) of the order $E \sim R^{-1}$. They correspond to changes of the state of motion of the constituent quarks over distances $\sim R$, which do not affect the internal structure of the constituent quark at distances $\sim \rho$. Excitations of energy $E \sim \rho^{-1}$ belong to the nonresonant hadronic continuum. Switching to the QCD language, our arguments of Section II show that the twist-2 quark distribution (here, the axial coupling, $g_{A}$ ) arises mainly from field configurations with energies/momenta of the order $R^{-1}$, while the twist-4 quark-gluon correlations are dominated by energies/momenta of the order $\rho^{-1}$. Comparing the hadronic and the QCD description, we conclude that quark-hadron duality should "work" for the twist-2 part of the structure function (i.e., the $Q^{2}$-independent part) already when summing over hadronic excitations with energies $E \sim R^{-1}$, but for the twist-2 plus twist-4 part of the structure function (i.e., to accuracy $1 / Q^{2}$ ) only when summing over hadronic excitations with energies $E \sim \rho^{-1}$. In practice, this means that quark-hadron duality in the structure function to accuracy $1 / Q^{2}$ may require integration over a significantly larger duality interval than duality to accuracy $\left(Q^{2}\right)^{0}$. This needs to be taken into account when trying to extract 
higher-twist matrix elements from resonance-based parametrizations of the structure functions. To summarize, the two-scale picture makes a clear parametric distinction between resonance and higher-twist contributions to the structure function. This qualitative prediction is supported by the fact that the phenomenological twist- 4 contribution to $g_{1}$ (see Fig. 4) seems to be dominated by small values of $x$, below the resonance region.

The two-scale picture described here is close in spirit to the Ioffe-Burkert parametrization of the $Q^{2}$ dependence of the first moment of $g_{1}^{p}$ [48], in which the contribution from the $\Delta$ resonance is separated from the continuum, and the leading power corrections are associated with the continuum contribution. The characteristic mass scale governing the power corrections in this parametrization, $\mu^{2}=M_{\rho}^{2}$, is numerically close to value associated with the constituent quark size, $\rho^{-2} \sim(0.3 \mathrm{fm})^{-2}=(600 \mathrm{MeV})^{2}$. Also, the analogous parametrization for the Bjorken sum rule ( $p-$ $n$ ) gives negative sign of the twist-4 correction, in agreement with our qualitative prediction. However, the numerical value of the twist- 4 correction obtained from the IoffeBurkert parametrization is substantially larger than the instanton vacuum estimate, corresponding to $f_{2}^{u-d} \approx$ $-2.3 g_{A} \mu^{2}=1.7 \mathrm{GeV}^{2}$.

More generally, the two-scale picture of hadron structure allows one to draw some conclusions about global properties of the transition from high to low $Q^{2}$ in the nucleon spin structure functions (i.e., going beyond the leading $1 / Q^{2}$-corrections). Since the characteristic mass scale for the power corrections is set by the size of the constituent quark, one should expect the twist expansion to break down at momenta of the order $Q^{2} \sim \rho^{-2}$. For the extraction of the leading $\left(1 / Q^{2}\right)$-corrections from $\mathrm{QCD}$ fits to the data this implies that one should restrict oneself to the range $Q^{2} \gg \rho^{-2}$, where the leading term in the series dominates (in our fit presented in Section III, $Q^{2}>$ $1 \mathrm{GeV}^{2}$ ).

The arguments presented in this paper can also be extended to higher-twist corrections to unpolarized deepinelastic scattering. A new feature in the unpolarized case is the appearance of twist- 4 operators measuring quark-quark correlations ("four-quark operators"), in addition to quark-gluon correlations of the type encountered in the polarized structure functions. Within our two-scale picture, the quark-quark correlation matrix elements are of the order $R^{-2}$, and thus parametrically suppressed compared to the quark-gluon ones, which are of the order $\rho^{-2}$. This qualitative conclusion seems to be in agreement with a joint analysis of the twist- 4 corrections to $F_{2}$ and $F_{L}$, see Ref. [49] for details. The role of the size of the constituent quark in power corrections to unpolarized structure function moments was also discussed in a different approach in Ref. [50]. Finally, we note that our picture of higher-twist effects suggests a possible relation between power corrections to unpolarized and polarized structure functions, as found in the renormalon approach of Ref. [51]. This aspect deserves further study.

To summarize, we have argued that the leading power corrections to the nucleon spin structure functions are governed by the short-distance scale due to the spontaneous breaking of chiral symmetry - the size of the constituent quark. The qualitative statements following from this assumption are supported by the result of a QCD fit to the present polarized DIS data. The arguments presented here may eventually serve as the basis for an "interpolating" model of the nucleon spin structure functions, connecting the scaling region at large $Q^{2}$ with the photoproduction point.

\section{ACKNOWLEDGMENTS}

We thank J. P. Chen, W. Melnitchouk, D. Stamenov, and M. Vanderhaeghen for useful discussions. This work is supported by U.S. Department of Energy Contract No. DE-AC05-84150, under which the Southeastern Universities Research Association (SURA) operates the Thomas Jefferson National Accelerator Facility. A. V.S. acknowledges financial support by RFBR (Grants No. 05-02-17748, No. 05-01-00992, No. 06-02-16215, and No. 06-02-81032) and the Heisenberg-Landau program.
[1] For a recent summary, see: D. Ryckbosch, in Proceedings of the 13th International Workshop on Deep Inelastic Scattering (DIS 2005), Madison, WI, 2005, edited by W. H. Smith and S.R. Dasu (American Institute of Physics, Melville, NY, 2005)

[2] E. Leader, A. V. Sidorov, and D. B. Stamenov, Phys. Rev. D 67, 074017 (2003); hep-ph/0309048.

[3] E. Leader, A. V. Sidorov, and D. B. Stamenov, hep-ph/ 0411323.

[4] E. V. Shuryak and A. I. Vainshtein, Nucl. Phys. B201, 141
(1982).

[5] X. D. Ji and P. Unrau, Phys. Lett. B 333, 228 (1994).

[6] B. Ehrnsperger, A. Schafer, and L. Mankiewicz, Phys. Lett. B 323, 439 (1994).

[7] Contrary to Ref. [6] and standard convention, we define the coefficient of the twist- 4 matrix element, $f_{2}$, Eq. (1), such that it has dimension (mass) ${ }^{2}$, i.e., we do not make it dimensionless by extracting in a factor of $M_{N}^{2}$ (nucleon mass). We shall see below that the value of $f_{2}$ is determined by a physical mass scale unrelated to the nucleon 
mass. Extracting a factor $M_{N}^{2}$ would obscure this fact, and burden the subsequent formulas with unnecessary factors.

[8] P. L. Anthony et al. (E155 Collaboration), Phys. Lett. B 553, 18 (2003).

[9] X. Zheng et al. (Jefferson Lab Hall A Collaboration), Phys. Rev. C 70, 065207 (2004).

[10] Z. E. Meziani et al., Phys. Lett. B 613, 148 (2005).

[11] M. Osipenko et al., Phys. Lett. B 609, 259 (2005).

[12] A. Deur et al., Phys. Rev. Lett. 93, 212001 (2004).

[13] A. Deur, nucl-ex/0508022.

[14] J. P. Chen, A. Deur, and Z. E. Meziani, Mod. Phys. Lett. A 20, 2745 (2005).

[15] D. Diakonov, Prog. Part. Nucl. Phys. 51, 173 (2003).

[16] T. Schafer and E. V. Shuryak, Rev. Mod. Phys. 70, 323 (1998).

[17] J. Balla, M. V. Polyakov, and C. Weiss, Nucl. Phys. B510, 327 (1998).

[18] N. Y. Lee, K. Goeke, and C. Weiss, Phys. Rev. D 65, 054008 (2002).

[19] M. Gockeler et al., Phys. Rev. D 63, 074506 (2001).

[20] X. Zheng et al. (Jefferson Lab Hall A Collaboration), Phys. Rev. Lett. 92, 012004 (2004).

[21] E. S. Ageev et al. (COMPASS Collaboration), Phys. Lett. B 612, 154 (2005).

[22] M. Kremer and G. Schierholz, Phys. Lett. B 194, 283 (1987).

[23] T. W. Chiu and T. H. Hsieh, Nucl. Phys. B673, 217 (2003); Nucl. Phys. B, Proc. Suppl. 129, 492 (2004).

[24] T. Doi, N. Ishii, M. Oka, and H. Suganuma, Phys. Rev. D 67, 054504 (2003).

[25] M. V. Polyakov and C. Weiss, Phys. Lett. B 387, 841 (1996).

[26] D. Diakonov and M. I. Eides, Pis'ma Zh. Eksp. Teor. Fiz. 38, 358 (1983) [JETP Lett. 38, 433 (1983)].

[27] D. Diakonov and V. Y. Petrov, Nucl. Phys. B272, 457 (1986).

[28] D. Diakonov, V. Y. Petrov, and P. V. Pobylitsa, Nucl. Phys. B306, 809 (1988).

[29] Here $k$ denotes the Minkowskian 4-momentum (all our formulas assume the Minkowskian metric). The integration effectively extends over spacelike (Euclidean) mo- menta, for which $-k^{2}=k_{\text {Euclid }}^{2}>0$.

[30] G. Martinelli and C. T. Sachrajda, Nucl. Phys. B478, 660 (1996).

[31] I. I. Balitsky, V. M. Braun, and A. V. Kolesnichenko, Phys. Lett. B 242, 245 (1990); 318, 648(E) (1993).

[32] E. Stein, P. Gornicki, L. Mankiewicz, and A. Schafer, Phys. Lett. B 353, 107 (1995).

[33] B. L. Ioffe, Yad. Fiz. 60N10, 1866 (1997) [Phys. At. Nucl. 60, 1707 (1997)].

[34] S. Wandzura and F. Wilczek, Phys. Lett. B 72, 195 (1977).

[35] E. Leader, A. V. Sidorov, and D. B. Stamenov, J. High Energy Phys. 06 (2005) 033.

[36] A. V. Sidorov and C. Weiss, hep-ph/0410253.

[37] C. Weiskopf, DESY Report No. DESY-THESIS-2002039.

[38] E. Leader, A. V. Sidorov, and D. B. Stamenov, Phys. Rev. D 73, 034023 (2006).

[39] A. Airapetian et al. (HERMES Collaboration), Phys. Rev. D 71, 012003 (2005).

[40] C. W. Kao, D. Drechsel, S. Kamalov, and M. Vanderhaeghen, Phys. Rev. D 69, 056004 (2004).

[41] X. D. Ji and W. Melnitchouk, Phys. Rev. D 56, R1 (1997).

[42] J. Edelmann, G. Piller, N. Kaiser, and W. Weise, Nucl. Phys. A665, 125 (2000).

[43] L. Frankfurt, M. Strikman, and C. Weiss, Ann. Phys. (Berlin). 13, 665 (2004).

[44] R. K. Ellis, W. Furmanski, and R. Petronzio, Nucl. Phys. B207, 1 (1982); B212, 29 (1983).

[45] X. D. Ji, Nucl. Phys. B402, 217 (1993).

[46] D. Diakonov, V. Petrov, P. Pobylitsa, M. V. Polyakov, and C. Weiss, Nucl. Phys. B480, 341 (1996); Phys. Rev. D 56, 4069 (1997).

[47] A. E. Dorokhov and N. I. Kochelev, Phys. Lett. B 304, 167 (1993).

[48] V.D. Burkert and B.L. Ioffe, Phys. Lett. B 296, 223 (1992).

[49] B. Dressler, M. Maul, and C. Weiss, Nucl. Phys. B578, 293 (2000).

[50] R. Petronzio, S. Simula, and G. Ricco, Phys. Rev. D 67, 094004 (2003); 68, 099901 (2003).

[51] A. L. Kataev, Mod. Phys. Lett. A 20, 2007 (2005). 